Autorização concedida ao Repositório Institucional da Universidade de Brasília (RIUnB) pelo Professor Kleber Melo e Silva, em 31 de janeiro de 2019, para disponibilizar o trabalho, gratuitamente, para fins de leitura, impressão e/ou download, a título de divulgação da obra. 


\title{
A Wavelet-Based Busbar Differential Protection
}

\author{
K. M. Silva, Member, IEEE, A. M. P. Escudreo, F. V. Lopes, Member, IEEE, and F. B. Costa, Member, IEEE
}

\begin{abstract}
This paper presents a new wavelet-based busbar differential protection algorithm, named as 87BW function. The instantaneous current-based differential protection fundamentals are taken into account and the well-known 1-out-of-1 and 2out-of-2 tripping logics are mapped into the wavelet domain. Operating and restraint wavelet coefficients energy of currents are computed, regarding station configuration and bus zones boundaries. Thereby, the proposed 87BW function can be properly used in both static and dynamic busbar configuration. Its performance was evaluated through EMTP-based simulations of faults in a $230 \mathrm{kV}$ power substation with double bus single breaker configuration. The obtained results reveal it provides ultra-high-fast trip for internal and evolving external-to-internal faults, guaranteeing security for normal through-load and external faults as well, even in the case of early and severe current transformer (CT) saturation.
\end{abstract}

Index Terms-Protective relaying, busbar differential protection, wavelet transform.

\section{INTRODUCTION}

A MONG faults in electric power apparatus, those in busbars are rare, accounting for only $6-7 \%$ of the total number of faults [1]. Even so, this kind of fault is cause of great concern for utilities, as it typically leads to high current levels and results in large disruption of loads and generations [2]. Therefore, the use of modern protection systems is of utmost importance to provide fast and selective busbar fault clearance, preserving the power system stability margins [3]. Therefore, researchers worldwide have made efforts to develop reliable protection functions able to provide fast and selective busbar fault clearance.

Power system busbars vary significantly in relation to their size (number of bays), complexity (number of sections, tiebreakers, disconnects, etc) and rated voltage (transmission and distribution) [4]. In large power substations, busbars are usually divided into bus zones. Typically, both high and low impedance differential functions have been used for busbar protection. High impedance element can deal with CT saturation quite effectively, but due to special $\mathrm{CT}$ requirements and physical wiring connection, its use is limited to static bus configuration only. On the other hand, nowadays, modern low impedance differential relays have been widely used for both static and dynamic busbar configurations, since it can easily handle with dynamic zone reconfiguration and with multiple

This research was supported in part by the Brazilian National Council for Scientific and Technological Development $(\mathrm{CNPq})$ and in part by the Brazilian Coordination for Improvement of Higher Education Personnel (Capes).

K. M. Silva, A. M. P. Escudero and F. V. Lopes are with the Department of Electrical Engineering at University of Brasília (UnB), 70910900 Brasília, DF, Brazil (e-mail: klebermelo@unb.br, ampianet@aluno.unb.br, felipevlopes@unb.br).

F. B. Costa is with Federal University of Rio Grande do Norte (UFRN), School of Science and Technology, Natal 59078-970, Brazil (e-mail: flaviocosta@ect.ufrn.br).
CT ratios, as well as it can be used in both centralized and distributed bus protection schemes straightforwardly [5], [6].

The advent of numerical relays has led to improvements in the traditional busbar differential protection (ANSI code 87B) [7]-[10]. In [7], the graph theory is used to introduce a new method to select bus zones. In [8], a reliable algorithm that includes busbar protection and advanced zone selection is described, by means of several protection principles and measuring techniques which use both instantaneous and phasor quantities. In [10], the differential protection algorithm is combined with a CT secondary current compensating scheme.

The fault clearing time can be divided into the protection scheme operating time (delays caused by the protective relays, communications channels, and auxiliary relays) and the fault current interruption time (breaker opening and arcing times). Aiming to speed up the fault clearance, manufacturers have made efforts on reducing relay tripping time instead of proposing new arc extinction apparatus for circuit breakers [11].

In respect to busbar, some algorithms based on wavelet transform have been reported as alternatives to reduce the protection scheme operating time [12]-[18]. In [12], a busbar differential protection scheme based on the Morlet wavelet is introduced, showing good performance during saturation and ratio mismatch of CTs. A similar method using wavelet packet transform is reported in [13]. In [14], it is proposed a waveletbased directional algorithm to distinguish in-zone from out-ofzone faults. In [15], fault indexes based on wavelet coefficients are compared with threshold values to distinguish between internal and external faults. Differential algorithms based on Mexican Hat and Meyer wavelet functions are reported in [16] and [17], respectively, presenting good results even in the case of CT saturation. In [18], a differential scheme based on continuous wavelet transform is reported, performing well, including for faults with severe CT saturation.

Undoubtedly, the wavelet-based busbar protection algorithms reported in [12]-[18] could provide very fast operating time for most internal faults and prevent false trip for external ones, even if CT saturation takes place. However, none of them is able to properly deal with evolving external-tointernal faults. As a result, their use in real-world protection applications can be limited.

In order to overcome the aforementioned drawbacks, this paper presents a new wavelet-based low-impedance busbar differential protection algorithm (87BW function), by means of mapping instantaneous current-based differential protection fundamentals into the wavelet domain. Thereby, the wellknown 1-out-of-1 and 2-out-of-2 tripping logics were adapted to perform with operating and restraint wavelet coefficients energy of currents, regarding station configuration and bus zones boundaries (dynamic bus replica). As a result, the proposed 87BW function can be properly used in both static 
and dynamic busbar configuration. Aiming to evaluate its performance, EMTP-based simulations of fault in a $230 \mathrm{kV}$ power substation with double bus single breaker configuration were carried out. The obtained results reveal the proposed protection function provides ultra-high-fast trip for internal and evolving external-to-internal faults, guaranteeing security for normal through-load and external faults as well, even in the case of early and severe CT saturation.

\section{Busbar Differential Protection Fundamentals}

The low-impedance busbar differential protection function can be implemented using either current phasors or current samples [3]. In the first case, dynamic slope adjustments can be applied to guarantee security for external faults with CT saturation [4]. Nevertheless, since phasors are used, the trip command takes a little longer to be issued (typically one cycle) [11]. Conversely, instantaneous-current based differential protection function provides some advantages, such as fast trip for internal faults (ranging from one quarter-cycle to half-cycle) and security for external faults with CT saturation, by using the well-known tripping logics 1-out-of-1 and 2-out-of-2 [2].

The instantaneous-current based differential protection function compares the operating current $\left(i_{o p}\right)$ with the restraint current $\left(i_{\text {res }}\right)$ to detect in-zone faults [2]:

$$
i_{\text {op }}=\left|\sum_{p=1}^{P} i_{p}\right| \quad i_{\text {res }}=\sum_{p=1}^{P}\left|i_{p}\right|,
$$

where $P$ is the number of network elements connected to the busbar and $i_{p}$ is the instantaneous current of the $p$-th element.

Ideally, the relay must operate whenever $i_{o p}$ exceeds a minimum operational current $\left(i_{\min }\right)$ and a operational slope $(S L P)$ multiplied by a smoothed version of $i_{\text {res }}$, named $\bar{i}_{\text {res }}$ :

$$
i_{o p}>i_{m i n} \text { AND } i_{o p}>S L P \cdot \bar{i}_{r e s}
$$

where $\bar{i}_{\text {res }}$ is obtained through a low-pass filtering process, what improves the protection security for external faults with CT saturation [2], [19].

In traditional busbar relays, $i_{\min }$ is defined by taking into account either the minimum short-circuit current of the busbar (considering the network development for the following years multiplied by a safety factor) or the maximum CT ratio of all substation bays (accounting for an open CT on any bay) [2]. The operational slope $S L P$ is included to account for CT errors and saturation during external faults [5].

Aiming to improve the protection scheme reliability, the trip command is issued only if the operating condition (2) is fulfilled during a certain period of time, typically chosen lesser than the CT time to saturate, for example, a quarter-cycle [2]. The time to saturate is a requirement that must be taken into account for CT selection. Therefore, the well-known tripping logics 1-out-of-1 and 2-out-of-2 are performed. Basically, it is counted how many times (2) is fulfilled during consecutive quarter-cycle periods, beginning just after $i_{o p}$ becomes greater than $S L P \cdot \bar{i}_{\text {res }}$. Thus, if there is one count soon after the fault inception (i.e., operating conditions is fulfilled during one quarter-cycle after the fault inception), a trip command is

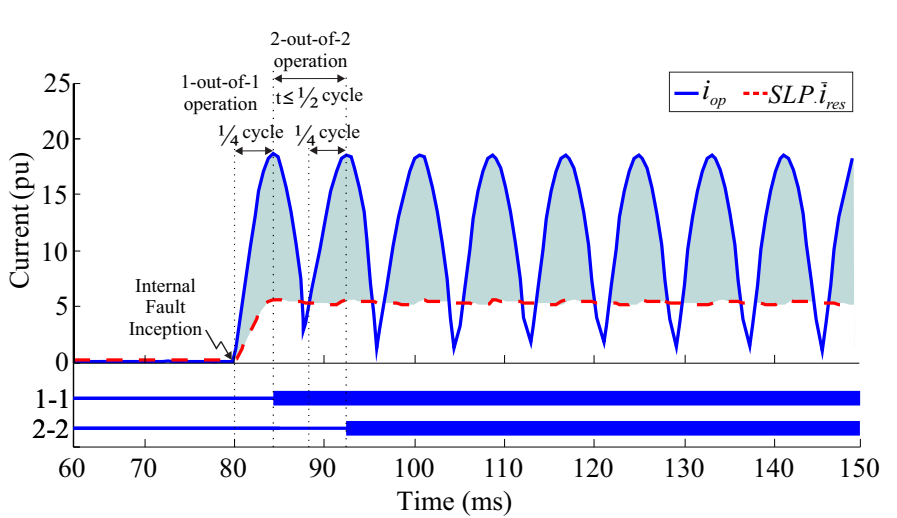

(a)

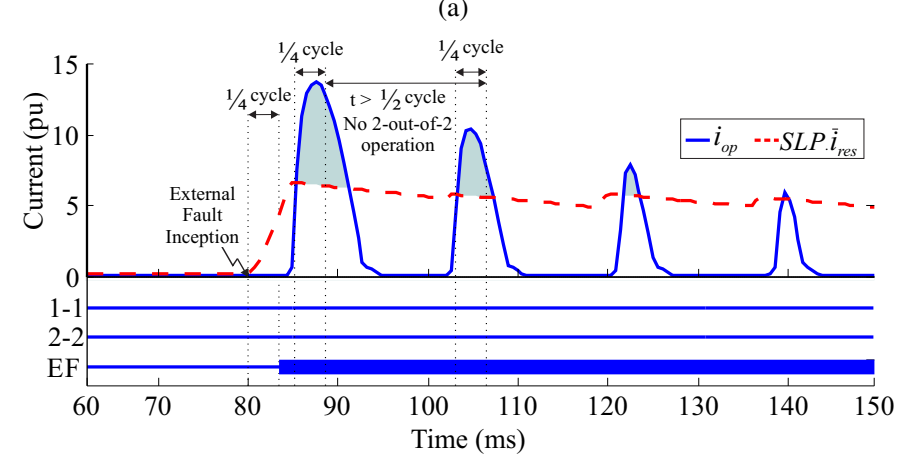

(b)

Fig. 1. Tripping logic evaluation for: (a) an internal fault; (b) an external fault with CT saturation.

issued by the 1-out-of-1 logic (fast operation mode). On the other hand, during evolving external-to-internal faults, if two counts are spaced by half-cycle, the trip command is issued using the 2-out-of-2 logic (secure operation mode). Fig. 1(a) illustrates the behavior of $i_{o p}, S L P \cdot \bar{i}_{\text {res }}$ and the 1-out-of-1 and 2-out-of-2 logics for an internal fault.

For external faults, $\bar{i}_{r e s}$ quickly increases, whereas $i_{o p}$ remains close to zero (Fig. 1(b)). If this condition remains during a period of time lesser than the time spent to CT saturates, an external fault is detected, toggling the state of an external fault flag $E F$. Thereby, the fast operation mode is blocked for a period of time (secure operation mode), typically set to $150 \mathrm{~ms}$ [2]. Thus, the trip command is only issued by the 2-out-of-2 logic, increasing tremendously the security during external faults with CT saturation, besides allowing properly operation for evolving external-to-internal faults.

An external fault with CT saturation is shown in Fig 1(b). Even with the increase of $i_{o p}$ due to the CT saturation, no false trip command is issued. Since the fast operation mode is blocked and there are no double counts spaced by a time lesser than half-cycle, the 2-out-of-2 logic is not fulfilled.

\section{Proposed 87BW FunCtion}

The flowchart diagram shown in Fig. 2 summarizes the overall process of the proposed 87BW function. The subscript $\phi$ represents the phase quantities, since low-impedance differential busbar protection is phase-segregated. Also, the subscript $1, \cdots, P$ denotes the number of network elements connected to the busbar. Basically, the current signals measured by CTs are normalized to compensate ratio mismatch. Then, the maximum 


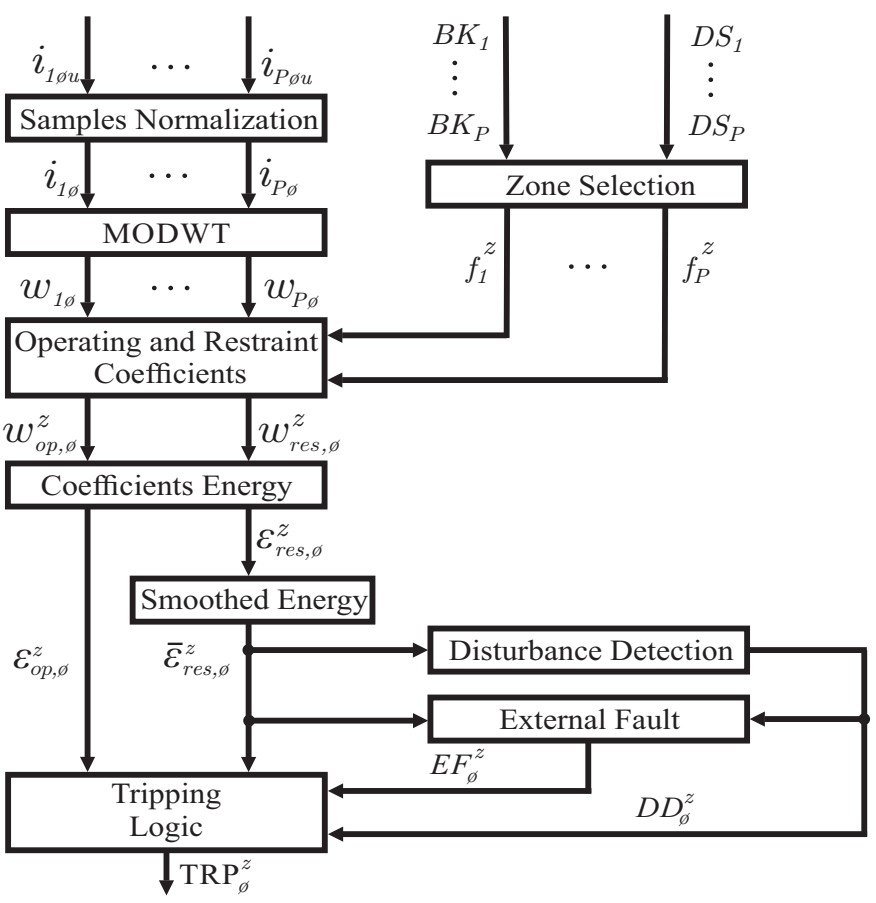

Fig. 2. Flowchart of the proposed 87BW function.

overlap discrete wavelet transform (MODWT) is performed to compute the wavelet coefficients. Based on the status of circuit breakers and disconnect switches, bus zone selection is performed and operating and restraint wavelet coefficients are obtained for each bus zone. After that, both operating and restraint wavelet coefficients energy are calculated. The smoothed restraint energy are then computed. Finally, tripping logics along with disturbance and external fault detection schemes are appled to provide fast and secure operation. Further details about each module are presented next.

\section{A. Samples Normalization}

The proposed 87BW function receives instantaneous values of the secondary currents of CTs in each element connected to the busbar. Then, in order to compensate CTs ratio mismatch, those currents are normalized to per unit using the following normalization factor [4], [8]:

$$
T A P_{p}=\frac{C T R_{M A X} \cdot I_{N O M}}{C T R_{p}},
$$

where $C T R_{M A X}$ and $I_{N O M}$ are the highest CT ratio among network elements and its nominal secondary current, respectively; and $C T R_{p}$ is the CT ratio of the $p$-th element.

\section{B. $M O D W T$}

The MODWT is a modified version of the Discrete Wavelet Transform (DWT), with advantages such as: 1) it is time invariant; 2) it does not require downsampling process by 2 [20]. As a result, MODWT has shown to be better than the traditional DWT in real-time analysis of transient components, thereby it was chosen to extract fault-induced transient information from the monitored current waveform samples in the proposed 87BW function [21]. Here, the 87BW uses the first level wavelet coefficients $w_{p}$ of a current signal $i_{p}$, which are computed by means of the MODWT wavelet filter $\widetilde{h}$ as follows:

$$
w_{p}(k)=\sum_{l=1}^{L} h(l) i_{p}(k-L+l),
$$

where $h(l)$ stands for the coefficients of the MODWT wavelet filters [20]; and $L$ is the number of coefficients of the mother wavelet. In this work, the Daubechies mother wavelet with 4 coefficients (Db4) is used, which offers fast time response on transients detection and is suitable for real-time applications [22], [23]. Further consideration on choosing the mother wavelet is presented in Subsection III-I.

\section{Zone Selection}

The zone selection module performs the dynamic bus replica. Aiming to do so, flags $f_{p \phi}^{z}$ are obtained from the status of circuit breakers $\left(B K_{p}\right)$ and disconnecting switches $\left(D S_{p}\right)$ of all bays connected to busbar, and then evaluated to remove or include measured currents in a bus zone $z$ [4].

\section{Operating and Restraint Coefficients}

Likewise the instantaneous current-based 87B function, the operating wavelet coefficient $\left(w_{o p}\right)$ and the restraint wavelet coefficient $\left(w_{\text {res }}\right)$ are calculated at every sampling instant considering the adaptive zone selection as follows:

$$
\begin{aligned}
& w_{o p, \phi}^{z}(k)=\left|\sum_{p=1}^{P} f_{p \phi}^{z} \cdot w_{p \phi}(k)\right|, \\
& w_{r e s, \phi}^{z}(k)=\sum_{p=1}^{P} f_{p \phi}^{z} \cdot\left|w_{p \phi}(k)\right| .
\end{aligned}
$$

\section{E. Coefficients Energy}

Depending on the fault conditions (fault resistance, inception angle, location and type) and power system configuration (reflection and refraction indexes), $w_{o p}$ and $w_{\text {res }}$ present oscillatory behavior, changing their polarity and magnitude during the time. On the other hand, the energy of $w_{o p}$ and $w_{\text {res }}$ is cumulative and well-behaved, in such way that it provides safer operation of the proposed scheme.

The operating wavelet coefficients energy $\mathcal{E}_{o p}^{z}$ is computed for bus zone $z$ through a sliding windowing process applied to $w_{o p}^{z}$ as follows [21], [24]:

$$
\mathcal{E}_{o p, \phi}^{z}(k)=\sum_{q=k-Q+1}^{k}\left[w_{o p, \phi}^{z}(q)\right]^{2},
$$

where $Q$ is the sliding window length, chosen to properly perform the tripping logics in wavelet domain. The restraint wavelet coefficients energy $\mathcal{E}_{\text {res }}^{z}$ is obtained similarly. Thus, the operating conditions of 1-out-of-1 and 2-out-of-2 logics can be properly evaluated. Discussion regarding the choice of $Q$ used to compute the energy of the wavelet coefficients is presented in Subsection III-I. 


\section{F. Smoothed Restraint Energy}

Aiming to avoid the proposed 87BW function misoperation, $\mathcal{E}_{\text {res }, \phi}^{z}$ must maintain a stable behavior during external faults with CT saturation. In fact, in this case, $\mathcal{E}_{o p, \phi}^{z}$ remains with negligible values until CT saturation begins, when it may become greater than $S L P \cdot \mathcal{E}_{\text {res }, \phi}^{z}$, leading to false trip. Therefore, to overcome this drawback, the smoothing strategy reported in [2], [19] for instantaneous current-based 87B function was adapted to the wavelet domain. Thereby, the smoothed version $\overline{\mathcal{E}}_{\text {res, } \phi}^{z}$ of the restraint energy is obtained, performing a lowpass filtering process.

\section{G. Disturbance Detection}

Among several disturbance detection algorithms reported on the literature, the Disturbance Detection module implements the method reported in [25]. In order to do so, $\overline{\mathcal{E}}_{\text {res, } \phi}^{z}$ is alternatively used as input of the method, rather than the energy of Park's transformation coefficients as originally done. Therefore, whenever $\overline{\mathcal{E}}_{\text {res, } \phi}^{z}$ abruptly changes (e.g. due to switching transients, either internal or external faults, etc.), a disturbance is detected and the flag $D D_{\phi}^{z}$ is set to 1 (one).

\section{H. External Fault}

Whenever a disturbance takes place, $\overline{\mathcal{E}}_{\text {res, } \phi}^{z}$ quickly increases, whereas $\mathcal{E}_{o p, \phi}^{z}$ remains stable with negligible values. Based on that, in this work, if during the first $2 \mathrm{~ms}$ just after the disturbance is detected, an external fault is declared, setting the flag $E F_{\phi}^{z}$ to 1 (one). Thus, the secure operation mode is performed, blocking the logic 1-out-of-1 during $150 \mathrm{~ms}$.

\section{Tripping Logic}

The operating conditions described in (2) are mapped into the wavelet domain as follows:

$$
\mathcal{E}_{o p, \phi}^{z}>\mathcal{E}_{\text {min }} \quad \text { AND } \mathcal{E}_{o p, \phi}^{z}>S L P \cdot \overline{\mathcal{E}}_{r e s, \phi}^{z},
$$

where $\mathcal{E}_{\min }$ is a minimum energy threshold and $S L P$ is a operational slope.

Different from traditional protection functions, variables such as minimum short-circuit current are not required for setting procedures of the proposed algorithm. In real world protection applications, $\mathcal{E}_{\min }$ could be set based on the analysis of the random nature of noise to ensure the security of the protection scheme as whole, as reported in [26]. On the other hand, $S L P$ must be set to guarantee sensibility to properly detect the two first incident traveling waves, in such way that large $S L P$ may lead the 1-of-of-1 and 2-out-of-2 tripping logics to misoperate.

As illustrated in Fig. 3, during an internal fault, $\mathcal{E}_{o p, \phi}^{z}$ and $\overline{\mathcal{E}}_{\text {res }, \phi}^{z}$ perform similarly to $i_{o p}$ and $\bar{i}_{\text {res }}$ in instantaneous current-based 87B function (see Section II), such that the well-known tripping logics 1-out-of-1 and 2-out-of-2 can be mapped into the wavelet domain effortlessly. Actually, a count is done whenever the operating conditions in (8) are fulfilled during a time interval $L \Delta t$ (where $\Delta t$ is the sampling interval), beginning as soon as $\mathcal{E}_{o p, \phi}^{z}>S L P \cdot \overline{\mathcal{E}}_{r e s, \phi}^{z}$. Thus, if the first count occurs just after the fault inception, a trip command is issued by the 1-out-of-1 logic (fast operation mode). On the

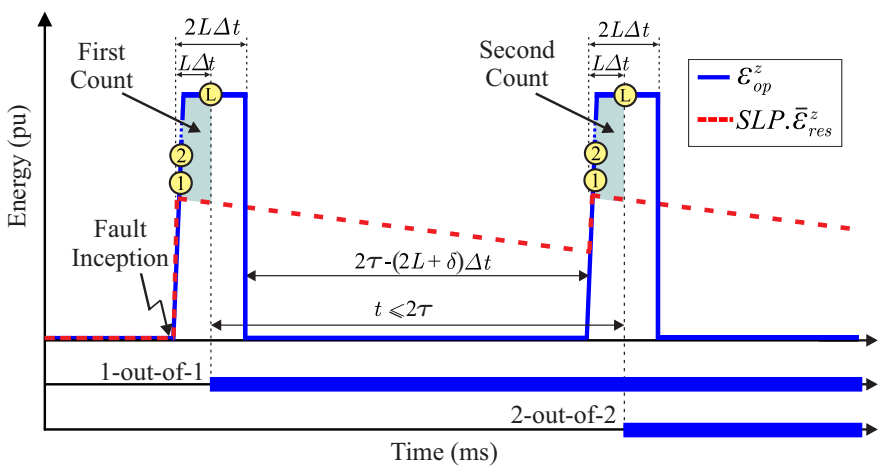

Fig. 3. Evaluation of the proposed 87BW tripping logics.

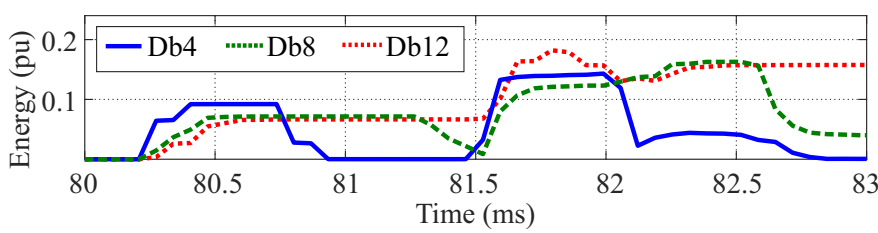

(a)

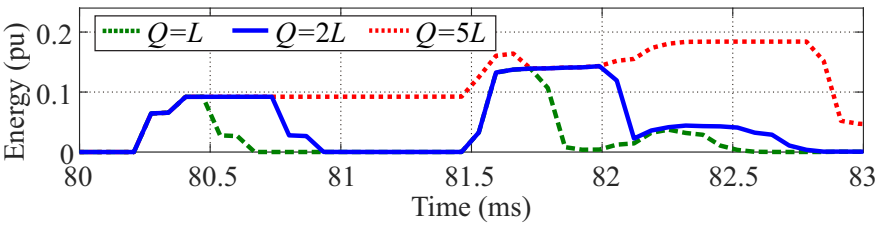

(b)

Fig. 4. Example of $\mathcal{E}_{o p, \phi}^{z}$ computed for: (a) different mother wavelets; different values of the data window length $Q$.

other hand, in the case of evolving external-to-internal faults, the 1-out-of-1 logic is blocked (see Section III-H), and a trip command is issued by the 2-out-of-2 logic if it is identified two successive counts spaced by $2 \tau$ (see Fig. 3), where $\tau$ is the propagation time of the smallest line connected to busbar.

As one can see in Fig. 3, to provide the proper operation of the 2-out-of-2 logic in the wavelet domain, it is necessary to detect two successive pulse in $\mathcal{E}_{o p}^{z}$ spaced by $2 \tau-(2 L+\delta) \Delta t$, where $\delta$ is a safety margin, because the behavior of $\mathcal{E}_{o p}^{z}$ may be different for each fault. It reveals that there is a trade-off in the choice of the following parameters: the mother wavelet; the data window length $Q$ used to compute the wavelet coefficients energy; and the sampling frequency $f_{s}$.

In Fig. 4(a), it is shown an example of $\mathcal{E}_{o p, \phi}^{z}$ computed for different mother wavelets of Daubechies family, considering the same $f_{s}$ and $Q=2 L$. The two successive pulses in $\mathcal{E}_{o p, \phi}^{z}$ can be lost for large mother wavelets, making the use of the 2out-of-2 logic unfeasible. In order to overcome this drawback, it is necessary either to reduce $Q$ or to increase $f_{s}$. However, as depicted in Fig. 4(b), if $Q>2 L$ the two successive pulses may be close enough to be lost, and if $Q<2 L$ they may be very narrow, so that they may not be detected. On the other hand, the requirement of very high $f_{s}$ may be not desired. That is the why the choice here is to use the mother wavelet Db4, providing fast and secure response on transients detection [22], [23]. Also, by using $Q=2 L$, the fast operation mode is guaranteed, as shown in Fig. 3. Furthermore, the minimum sampling frequency $f_{s, \text { min }}$ to fulfill the Nyquist's theorem 
and to perform the 2-out-of-2 logic in the wavelet domain is obtained as follows:

$$
f_{s, \min }=\frac{2 L+\delta}{2 \tau} .
$$

For example, considering $\delta=L$ and the smallest transmission lines connected to busbar with $180 \mathrm{~km}$ long $(\tau=0.6 \mathrm{~ms}$ for lossless lines), so $f_{s, \min }$ is $10 \mathrm{kHz}$.

As one can see, depending on the length of the smallest line connected to the busbar, high sampling frequency is required to implement the 2-out-of-2 logic. Nevertheless, after years of steady development, modern electronic components have reached the processing power levels necessary to implement even the most demanding protection algorithms, so that the use of high sampling frequency (in the order of $\mathrm{MHz}$ ) has not been an application limit for protective relaying anymore [11].

It is noteworthy to point out that, even though the proposed tripping logics are based on the traditional ones, their basis are completely different. The traditional 1-out-of-1 and 2-out-of2 tripping logics focused on the behavior of the fundamental frequency component [2]. On the other hand, the proposed tripping logics focused mainly on the detection of the time between the two first incident traveling waves.

\section{Analyzed Power System Model}

Fig. 5 shows the single line diagram built in the software ATPDraw for the $230 \mathrm{kV} / 60 \mathrm{~Hz}$ power system model evaluated in this paper. It comprises a substation with double bus single breaker arrangement, in which four transmission lines $180 \mathrm{~km}$ long (TL1, TL2, TL3 e TL4) and two power transformers (TF1 and TF2) are connected. During normal operating conditions, TL1, TL3 and TF1 are connected to Bus 1, whereas TL2, TL4 and TF2 are connected to Bus 2, and Buses 1 and 2 are connected through the bus coupler circuit breaker (CB). The transmission lines were modeled as perfectly transposed with distributed and frequency-independent parameters, whereas power transformers were modeled using the saturable transformer model. Their electrical parameters were taken from actual power apparatus of the Brazilian power system, which are shown in Tables I and II. Remote buses are connected to Thvenin equivalent circuits, whose parameters are shown in Table III. The models used for CTs and coupling capacitors voltage transformers (CCVTs) are reported by the IEEE Power System Relaying Committee in [27].

\section{Performance Evaluation}

The proposed 87BW function was compared with the traditional instantaneous current-based 87B function using fault signals simulated in the $230 \mathrm{kV} / 60 \mathrm{~Hz}$ power system model shown in Fig. 5. To emulate the sampling process, the simulations were carried out using time-step of $0.1 \mu \mathrm{s}$. Then, current signals were filtered by an anti-aliasing third-order Butterworth filter with cutoff frequency at $7 \mathrm{kHz}$. Afterwards, the output signals were resampled using the sampling frequency $f_{s}=15360 \mathrm{~Hz}$. For the sake of simplicity, the proposed 87BW function takes into account the adaptive logics of bus zone selection for a double bus single breaker arrangement reported by the IEEE Power System Relaying Committee in [1]. The
TABLE I

TRANSMISSION LINES ELECTRICAL PARAMETERS

\begin{tabular}{cccc}
\hline$Z_{0}(\Omega / \mathrm{km})$ & $Z_{1}(\Omega / \mathrm{km})$ & $B_{0}(\mu S / \mathrm{km})$ & $B_{1}(\mu S / \mathrm{km})$ \\
\hline \hline $0.532+j 1.541$ & $0.098+j 0.510$ & 2.293 & 3.252 \\
\hline
\end{tabular}

TABLE II

POWER TRANSFORMER ELECTRICAL PARAMETERS

\begin{tabular}{ccc}
\hline Transformer & MVA & Rated Voltage $(\mathrm{kV})$ \\
\hline \hline TF1 & 450 & $500(\mathrm{Y}) / 230(\mathrm{Y}) / 13.8(\Delta)$ \\
TF2 & 150 & $230(\mathrm{Y}) / 69(\Delta) / 13.8(\Delta)$ \\
\hline
\end{tabular}

TABLE III

THÉVENIN POWER SYSTEM EQUIVALENTS

\begin{tabular}{cccc}
\hline Source & Voltage $(\mathrm{pu})$ & $Z_{0}(\Omega)$ & $Z_{1}(\Omega)$ \\
\hline \hline TL1 & $1.00 \angle 0^{\circ}$ & $16.07+j 25.04$ & $12.05+j 18.78$ \\
TL2 & $1.00 \angle-5^{\circ}$ & $18.41+j 28.69$ & $13.39+j 20.89$ \\
TF1 & $1.00 \angle-5^{\circ}$ & $28.07+j 43.74$ & $18.71+j 29.16$ \\
TF2 & $1.00 \angle 0^{\circ}$ & $5.52+j 8.61$ & $4.02+j 6.26$ \\
TL3 & $1.00 \angle-10^{\circ}$ & $18.42+j 28.69$ & $13.39+j 20.87$ \\
TL4 & $1.00 \angle 4^{\circ}$ & $16.07+j 25.04$ & $12.05+j 18.78$ \\
\hline
\end{tabular}

parameters $S L P$ and $\mathcal{E}_{\min }$ of the 87BW function were set to, respectively, 0.4 and $10^{-4}$ pu (based on the analysis of the energy of wavelet coefficients for a wide variety of fault scenarios), whereas $S L P$ and $I_{\min }$ of $87 \mathrm{~B}$ function were set to 0.25 and $0.1 \mathrm{pu}$, respectively. Some of the obtained results are presented next, but only faults on Bus 1 (zone $Z_{1}$ ) are covered, since the results for Bus 2 (zone $Z_{2}$ ) are similar. Also, the flags $D D$ and $E F$ are shown as the combination of those ones related to each phase of Bus 1. Firstly, the results for three case studies are presented: internal fault, external fault with CT saturation and evolving external-to-internal fault. Then, a massive data analysis was carried out, considering both internal and evolving faults.

\section{A. Case 1: Internal Fault}

A single-phase-to-ground fault in phase A (AG fault) was simulated at the Bus 1 at $82 \mathrm{~ms}$. The performance of the proposed 87B function is depicted in Fig. 6. As can be seen, a fault is detected and $\mathcal{E}_{o p, a}^{1}$ remains greater than $S L P \cdot \overline{\mathcal{E}}_{r e s, a}^{1}$ thenceforth, leading to trip command issuing in $0.26 \mathrm{~ms}$ after the fault inception by the 1-out-of-1 logic in the wavelet domain. Also, the 87BW function detects external fault in the healthy phases ( $\mathrm{B}$ and $\mathrm{C}$ ), enabling the secure operation mode of their differential elements. On the other hand, the performance of the 87B function for the same AG fault is shown in Fig. 7. In this case, the 1-out-of-1 logic issues a trip $4.1 \mathrm{~ms}$ after fault inception $(\approx 15 \times$ slower $)$. Also, likewise the proposed 87BW function, the traditional 87B function detects external fault in phases B and C, activating the secure operation mode for the correspondent differential elements.

\section{B. Case 2: External Fault with CT Saturation}

A close-in external three-phase fault was simulated at the line TL1, $1 \mathrm{~km}$ away from the Bus 1 . The performance of the proposed 87BW function is plotted in Fig. 8. It can be 


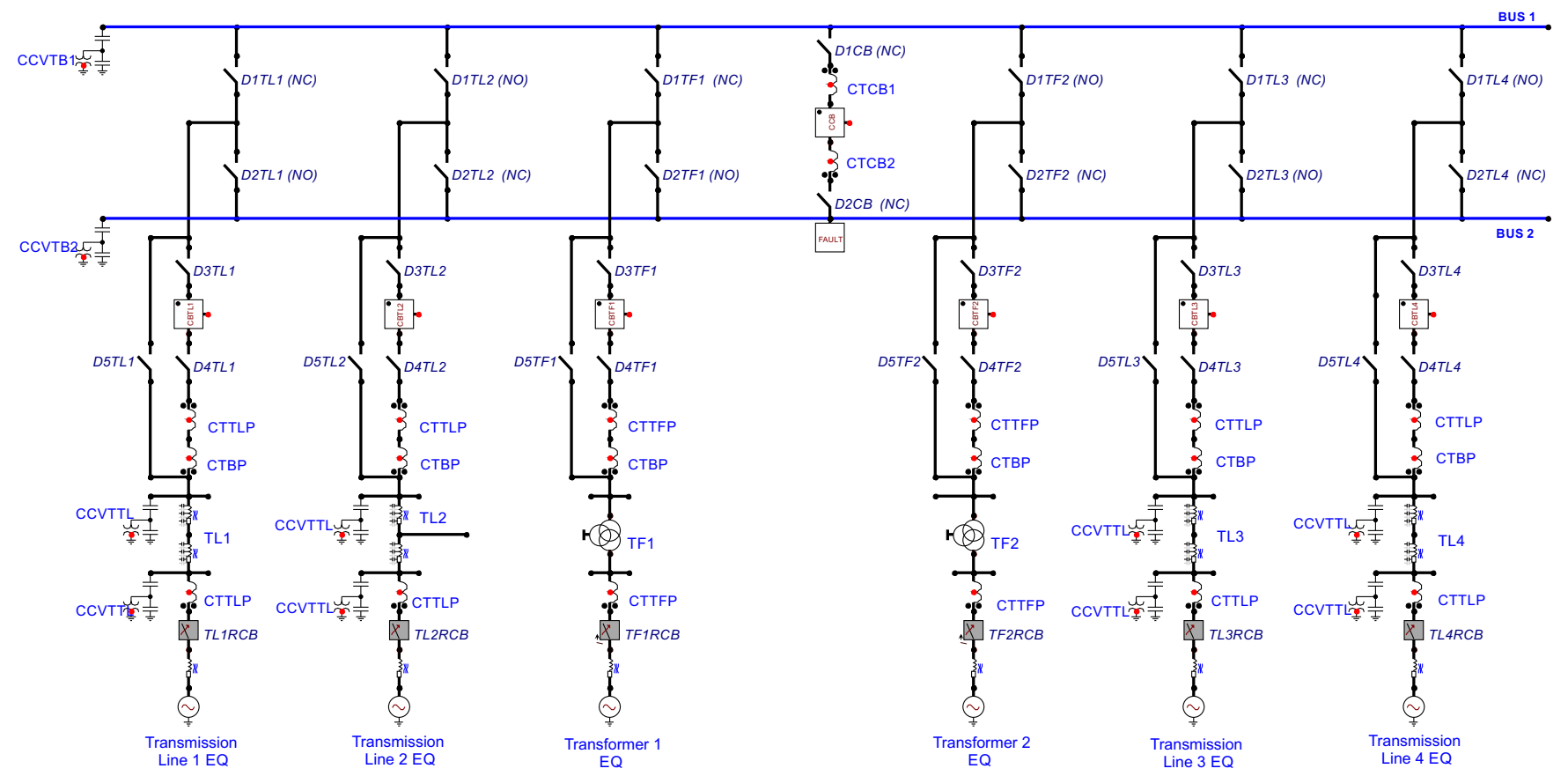

Fig. 5. Single-line diagram built in the ATPDraw of the analyzed power system model.

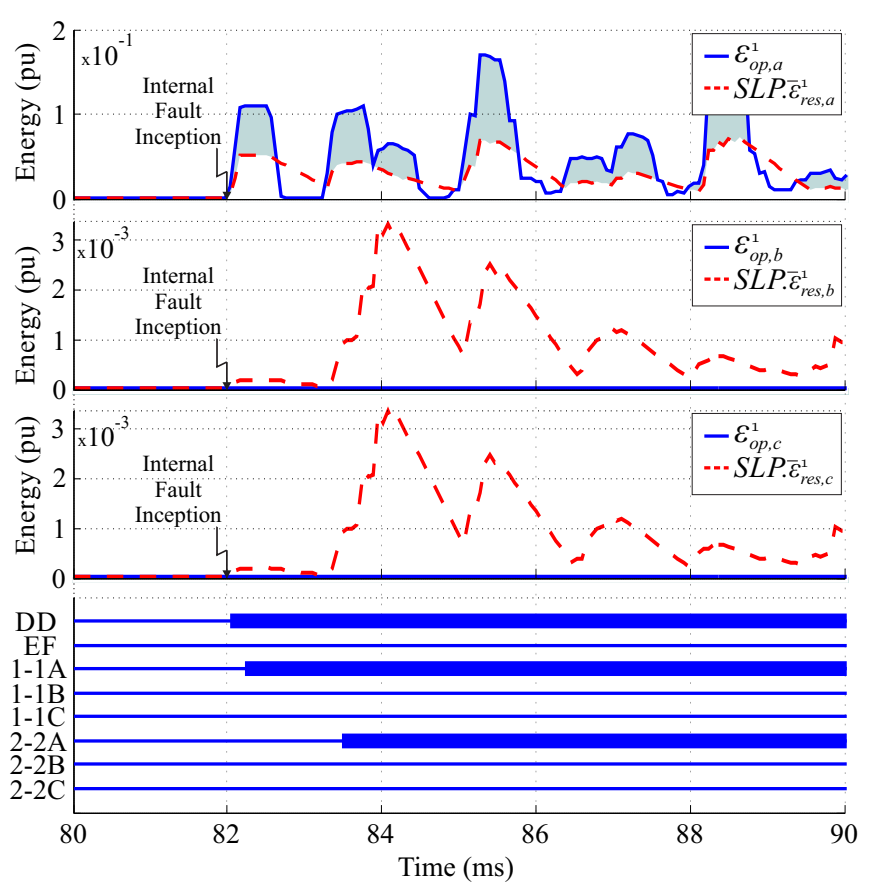

Fig. 6. The performance of the proposed 87BW function for the Case 1.

observed that when the fault takes place, only $\overline{\mathcal{E}}_{\text {res }}^{1}$ increases, whereas $\mathcal{E}_{o p}^{1}$ presents negligible values, leading to external fault detection. As can be seen, $\mathcal{E}_{o p}^{1}$ increases whenever a CT saturation period starts, but no false trip is issued by the 1-outof-1 logic, since it is disabled during external faults (secure operation mode). Actually, the 2-out-of-2 logic also does not issues a trip either, because there is no double counts spaced by a time lesser than $2 \tau$ (see Sec. III-I). As a result, the proposed 87BW function provides security during external faults with early and severe CT saturation. In this case, the traditional instantaneous current-based 87B function also performs well, since the secure operation mode is enabled (see Fig. 9).

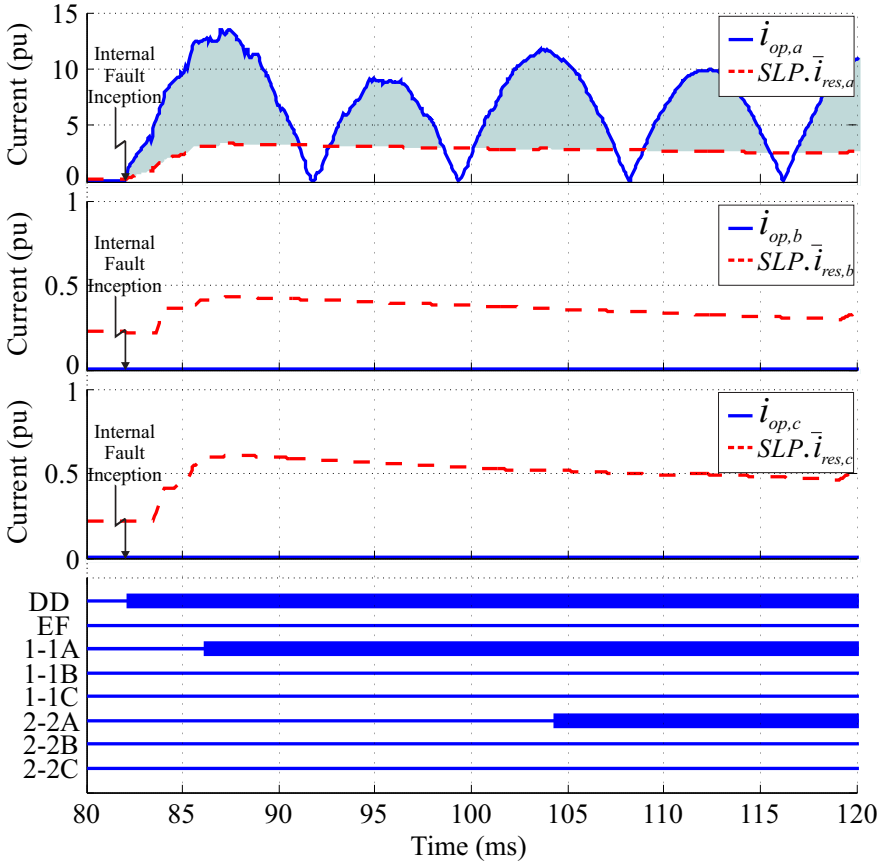

Fig. 7. The performance of the traditional 87B function for the Case 1.

Thereby, the 1-out-of-1 logic is blocked and there is no trip from the 2-out-of-2 logic, since there is no double counts spaced by half-cycle (see Sec. II). In fact, the saturation periods are spaced by a time greater than half-cycle [2].

\section{Case 3: Evolving External-to-Internal Fault}

Aiming to evaluate the proposed 87BW function for evolving faults, it is considered that a close-in AG external fault takes place at $80 \mathrm{~ms}$, leading to CT saturation. At $102 \mathrm{~ms}$, the external fault evolves to a phase-to-phase-to-ground internal fault between phases A and B (ABG fault). The performance of the 87BW function is shown in Fig. 10. The external fault 


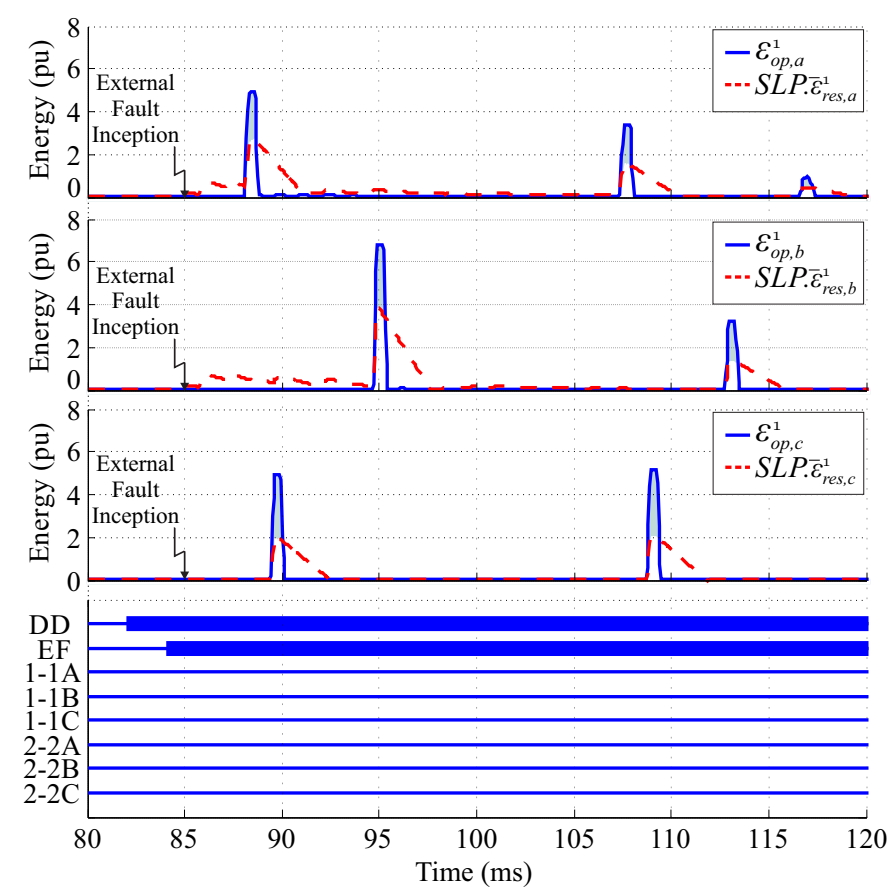

Fig. 8. The performance of the proposed 87BW function for the Case 2.

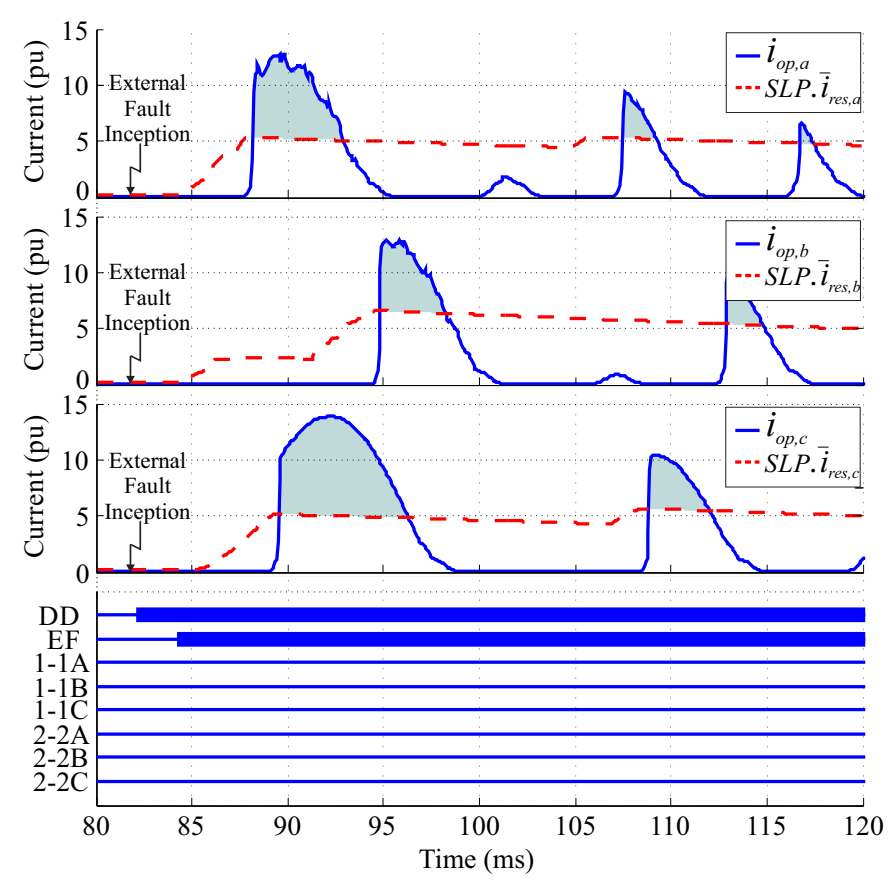

Fig. 9. The performance of the traditional 87B function for the Case 2 .

is detected and the secure operation mode is enabled, avoiding false trip when the CT on phase A saturates at $85 \mathrm{~ms}$. When the external fault evolves to the internal one, the differential element of phase B issues a trip $1.52 \mathrm{~ms}$ after the internal fault inception. On the other hand, the differential element of phase A sends a trip in $7.72 \mathrm{~ms}$, due to a hard transients attenuation. Even so, since three-phase trip is always used for busbar protection, the 87BW function issues a trip in $1.52 \mathrm{~ms}$. The performance of the 87B function is depicted in Fig. 11. As presented, the external fault is also detected. By the analysis of $i_{o p, a}$, it is observed that $87 \mathrm{~B}$ function is quite sensitive to

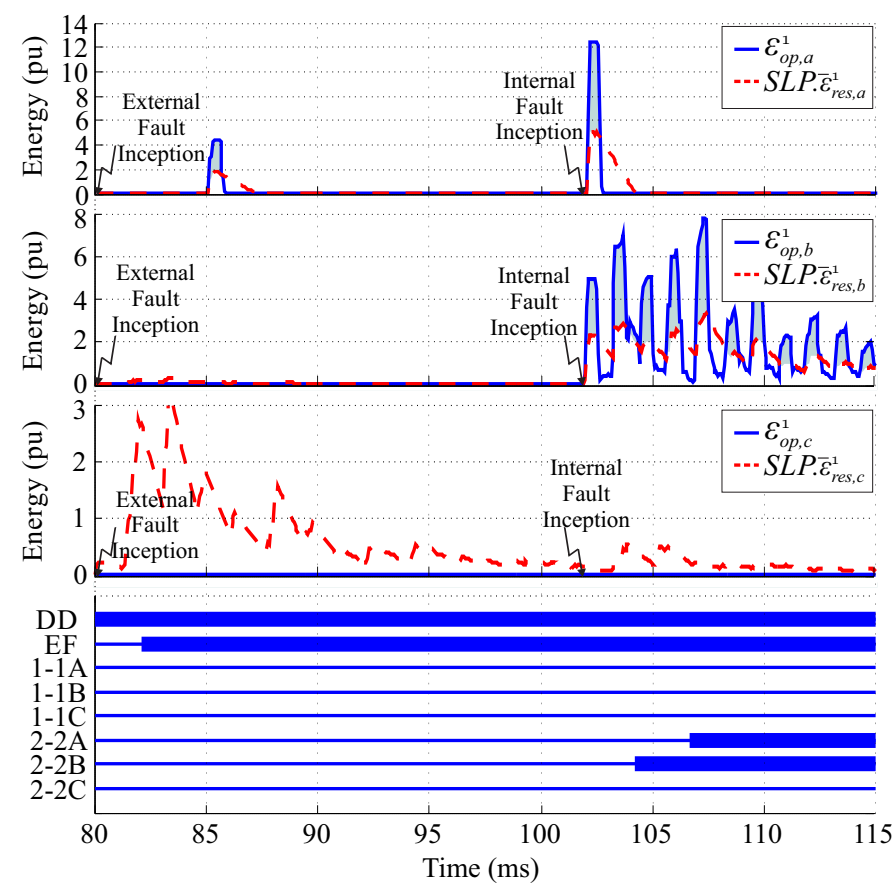

Fig. 10. The performance of the proposed 87BW function for the Case 3.
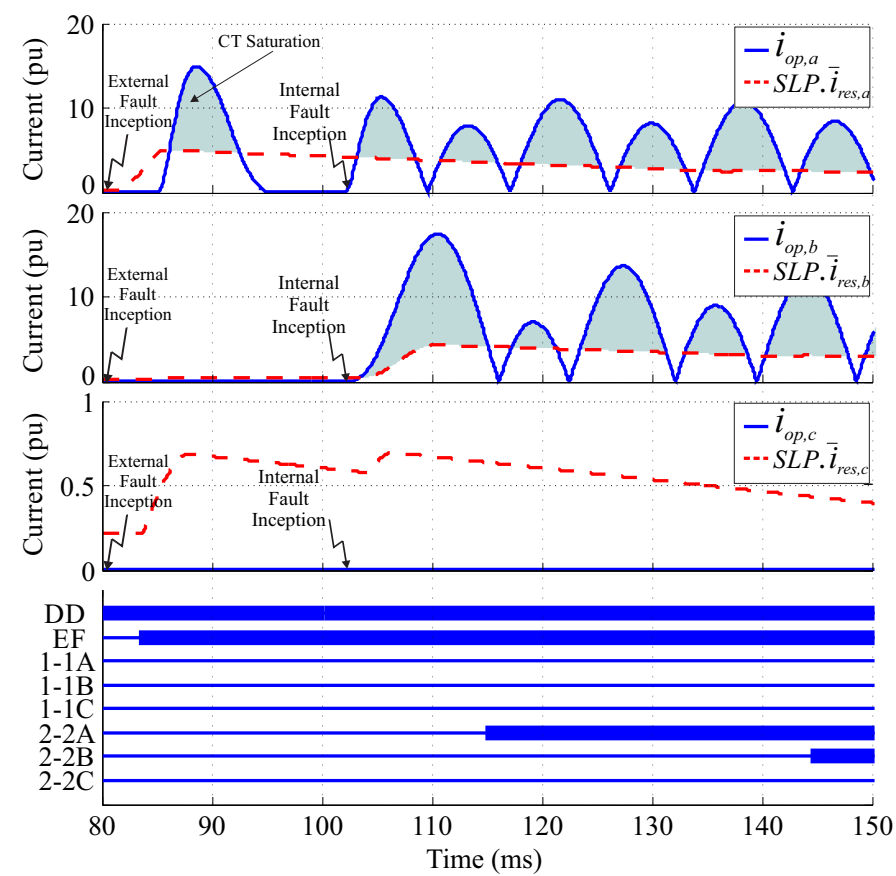

Fig. 11. The performance of the traditional 87B function for the Case 3 .

CT saturation. Even so, no false trip is issued, since the 1-outof-1 logic is blocked during external faults. The differential element of phase A send a trip $11.6 \mathrm{~ms}$ after the fault occurs $(\approx 7.5 \times$ slower than the $87 \mathrm{BW}$ function). Conversely, the differential element of the phase B issues a trip only 42.5 ms after the fault inception, due to a severe decaying dc component effect. Indeed, as the external fault evolves to the internal one, a high decaying dc component arises in the phase B current. As a result, the lobes of $i_{o p, b}$ alternate between high and low levels, delaying the double counting spaced by halfcycle required by the 2-out-of-2 logic (see Section II), such 
that the trip is only issued in the 5th lobe of $i_{o p, b}$ [2]. It is important to highlight that the proposed 87BW function does not suffer from the decaying dc component effect.

\section{Massive Data Analysis}

The performance of the evaluated protection functions were further evaluated through a massive data analysis. In order to do so, a wide variety of internal and evolving externalto-internal faults were simulated in the power system model shown in Fig. 5, by varying the fault resistance of ground faults $\left(R_{G}\right)$, the fault resistance of ungrounded faults $\left(R_{F}\right)$, the fault inception angle $(\theta)$ and the fault type, as described in Table IV. In the case of evolving faults, it was always considered that a close-in AG external fault with $R_{G}=150 \Omega$ evolves to an internal fault. As a whole, an overall of 350 internal faults and 350 evolving external-to-internal faults were simulated. Also, the noise effect on the evaluated protection functions was also evaluated, by adding to the simulated signals a white random noise with a signal-to-noise ratio (SNR) per sample of $50 \mathrm{~dB}$.

The operating time mean $(\mu)$ and standard deviation $(\sigma)$ of the evaluated protection functions are summarized in Table V. As it can be seen, the proposed 87BW function provides quite faster operating time than the 87B function, mainly for internal faults, for which the trip command is issued very fast by the 1-out-of-1 logic. On the other hand, in the case of evolving faults, the trip is a little bit slower, since it comes from the 2-out-of-2 logic. Nevertheless, it is still quite faster than the one provided by the 87B function.

In Fig. 12, it is shown the scatter plot of the operating times of the proposed 87BW function $\left(t_{87 B W}\right)$ versus the operating times of the traditional instantaneous current-based $87 \mathrm{~B}$ function $\left(t_{87 B}\right)$. It can be observed that the proposed $87 \mathrm{BW}$ function is always more than ten times faster than the 87B function for internal faults, whereas it is five times faster for most of evolving faults, except for some cases in which the AG external fault evolves to an AG internal one. In fact, it is noteworthy to point out that the proposed 87BW function did not operate for cases in which the AG external fault evolves to an $\mathrm{AG}$ internal fault with $R_{G}$ equal to 75 or $100 \Omega$. Actually, whenever a busbar external fault evolves to an internal one by taking the same phases, the transients signals are quite damped in those phases, which may be even more severe depending on the fault resistance and the fault inception angle. Moreover, though the proposed 87BW function has performed well even when noise is taking into account, one ought to bear in mind that in realworld applications, transient-based protection functions must be out of service whenever electromagnetic interference (EMI) noise can compromise relay security [28]. Conversely, despite having slower operating times, the traditional instantaneous current-based 87B function operates properly for all faults and has proven to be quite robust to noise. Therefore, the proposed 87BW function may be implemented along with the 87B function, providing tremendous fast operating times for most of busbar faults, while ensuring the protection security and reliability.
TABLE IV

FAULT PARAMETERS USED IN THE MASSIVE DATA ANALYSIS

\begin{tabular}{cl}
\hline Parameter & \multicolumn{1}{c}{ Value } \\
\hline \hline$R_{G}$ & $0,25,50,75$ and $100 \Omega$ \\
$R_{F}$ & $0,5,10,15$ and $20 \Omega$ \\
$\theta$ & $0^{\circ}, 30^{\circ}, 60^{\circ}, \ldots, 150^{\circ}$ and $180^{\circ}$ \\
Fault Type & $\mathrm{AG}, \mathrm{BG}, \mathrm{CG}, \mathrm{AB}, \mathrm{BC}, \mathrm{CA}, \mathrm{ABG}, \mathrm{BCG}, \mathrm{CAG}$ and $\mathrm{ABC}$ \\
\hline
\end{tabular}

TABLE V

STATISTICS OF THE OPERATING TIMES IN MILLISECONDS

\begin{tabular}{cccccc}
\hline \multirow{2}{*}{ Measure } & \multicolumn{2}{c}{ Internal Faults } & & \multicolumn{2}{c}{ Evolving Faults } \\
\cline { 2 - 3 } \cline { 5 - 6 } \cline { 5 - 6 } & Without Noise & With Noise & & Without Noise & With Noise \\
\hline \hline$\mu_{87 B W}$ & 0.2261 & 0.2197 & & 1.5443 & 1.5646 \\
$\sigma_{87 B W}$ & 0.0327 & 0.0818 & & 0.7346 & 0.7169 \\
\hline$\mu_{87 B}$ & 5.4416 & 5.3688 & & 12.9386 & 12.9187 \\
$\sigma_{87 B}$ & 1.9090 & 1.7423 & & 5.1792 & 5.1060 \\
\hline
\end{tabular}

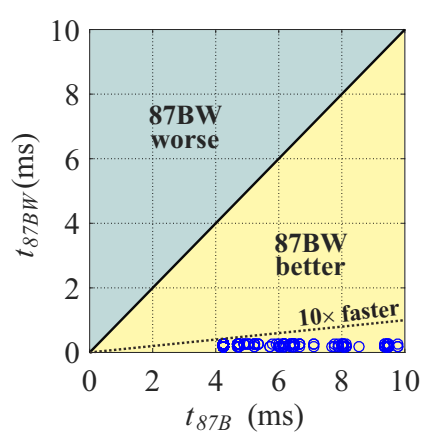

(a)

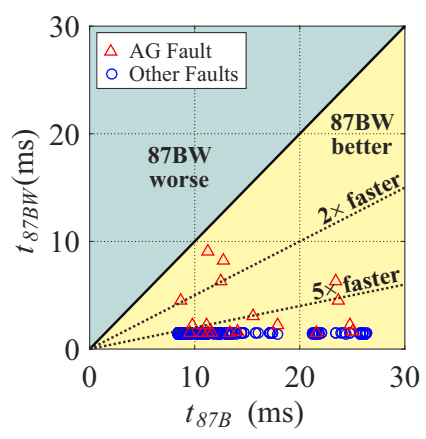

(c)

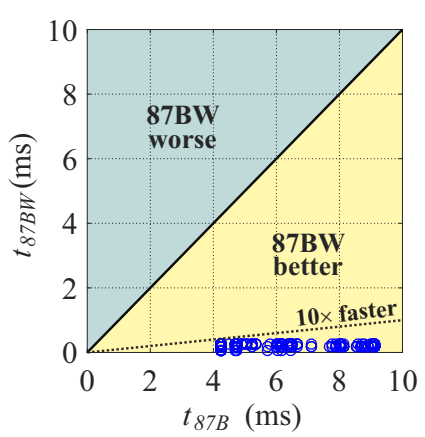

(b)

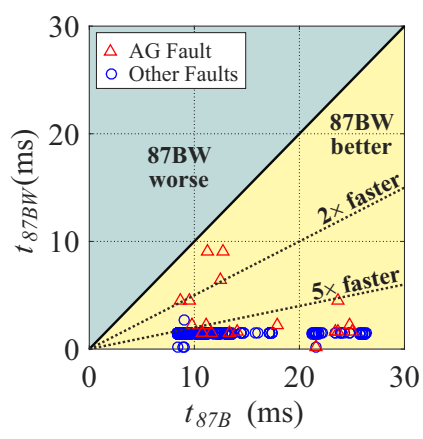

(d)
Fig. 12. Scatter plot of the operating times of the proposed 87BW function $\left(t_{87 B W}\right)$ and the current-based $87 \mathrm{~B}$ function $\left(t_{87 B}\right)$ : (a) internal faults without noise; (b) internal faults with noise; (c) evolving external-to-internal faults without noise; (d) evolving external-to-internal faults with noise.

\section{CONCLUSIONS}

An new wavelet-based low impedance busbar protection function was presented in this paper, by mapping the fundamentals of the instantaneous current-based 87B function to the wavelet domain. Unlike other algorithms reported in the literature, the proposed protection function provides ultra-highfast operating time for both internal and evolving externalto-internal busbar faults, while ensuring no false trip during external faults with early and severe CT saturation. Moreover, it considers dynamic bus replica, thereby it can be properly used in both static and dynamic busbar configuration. 
The obtained results reveal that the proposed 87BW function is more than ten times faster than the traditional 87B function for internal faults, and it is five times faster for most of evolving external-to-internal faults. Nevertheless, admittedly, the proposed 87BW function may not operate if fault-induced transients are over-damped. Therefore, the authors claim that in real-world applications, it may be implemented in conjunction with the traditional instantaneous current-based 87B function, providing ultra-high-fast operating times and guaranteeing the busbar protection security and reliability.

\section{REFERENCES}

[1] IEEE Std C37.234 - Guide for Protective Relay Applications to Power System Buses, IEEE Power \& Energy Society, Power System Relaying Committee, 2009.

[2] G. Ziegler, Numerical Differential Protection: Principles and Applications, 2nd ed. Berlin, Germany: Siemens, 2012.

[3] Cigré TB 431 - Modern Techniques for Protecting Busbars in HV Networks, Cigré Working Group B5.16, 2010.

[4] H. J. Altuve and E. O. Schweitzer, Modern Solutions for Protection, Control and Monitoring of Electric Power Systems. Pullman, USA: Schweitzer Engineering Laboratories, Inc., 2010.

[5] D. C. K. Behrendt and S. E. Zocholl, "Considerations for using highimpedance or low-impedance relays for bus differential protection," in 63rd Annual Conference for Protective Relay Engineers, Apr. 2010.

[6] C. Martin, S. Chase, T.-X. Nguyen, D. J. Hawaz, J. Pope, and C. Labuschagne, "Bus protection considerations for various bus types," in 40th Annual Western Protective Relay Conference, Atlanta, Georgia, USA, May 2013.

[7] A. G. B. Lin and E. O. Schweitzer, "A new method for protection zone selection in microprocessor-based bus relays," IEEE Transactions on Power Delivery, vol. 15, pp. 876-887, 2000.

[8] A. Guzmán, B.-L. Qin, and C. Labuschagne, "Reliable busbar protection with advanced zone selection," IEEE Transactions on Power Delivery, vol. 20, no. 2, pp. 625-629, Apr. 2005.

[9] Y. C. Kang, U.-J. Lim, S.-H. Kang, and P. A. Crossley, "A busbar differential protection relay suitable for use with measurement type current transformers," IEEE Transactions on Power Delivery, vol. 20, no. 2, pp. 1291-1298, Apr.

[10] Y. C. Kang, J. S. Yun, B. E. Lee, S. H. Kang, S. I. Jang, and Y. G. Kim, "Busbar differential protection in conjunction with a current transformer compensating algorithm," IET Generation, Transmissions \& Distribution, vol. 2, no. 1, pp. 100-109, 2008.

[11] E. O. Schweitzer, B. Kasztenny, A. Guzmn, V. Skendzic, and M. V. Mynam, "Speed of line protection - can we break free of phasor limitations?" in 2015 68th Annual Conference for Protective Relay Engineers, March 2015, pp. 448-461.

[12] M. M. Eissa, "A novel wavelet approach to busbar protection during ct saturation and ratio mismatch," Electric Power System Research, vol. 72, pp. 41-48, Jun. 2004

[13] M. M. Eissa, "High-speed differential busbar protection using waveletpacket transform," IEE Proceedings in Generation, Transmission \& Distribution, vol. 152, no. 6, pp. 927-933, Nov. 2005.

[14] S. P. Valsan and K. S. Swarup, "Computationally coefficient wavelettransform-based digital directional protection for busbars," IEEE Transactions on Power Delivery, vol. 22, no. 3, pp. 1342-1350, Jul. 2007.

[15] S. A. Gafoor and P. V. R. Rao, "A transient current based busbar protection scheme using wavelet transforms," International Journal of Electrical Power \& Energy Systems, vol. 33, pp. 1049-1053, 2011.

[16] M. M. Eissa, "Improvement of the differential busbar characteristic to avoid false operation during to ct saturation," IET Generation, Transmission \& Distribution, vol. 6, no. 10, pp. 931-939, 2012.

[17] M. M. Eissa, "A new digital busbar protection technique based on frequency information during ct saturation," International Journal of Electrical Power \& Energy Systems, vol. 45, pp. 42-49, 2013.

[18] M. M. Eissa, "New differential busbar characteristic based on high frequencies extracted from faulted signal during current transformer saturation," IET Generation, Transmission \& Distribution, vol. 8, pp. 619-628, 2014

[19] A. Kumar and P. Hansen, "Digital bus-zone protection," IEEE Computer Applications in Power, vol. 6, no. 4, pp. 29-34, Oct 1993.

[20] D. B. Percival and A. T. Walden, Wavelet Methods for Time Series Analysis. New York: Cambridge University Press., 2000.
[21] F. B. Costa, "Fault-induced transient detection based on real-time analysis of the wavelet coefficient energy," IEEE Transactions on Power Delivery, vol. 29, pp. 140-153, 2014.

[22] W. Wilkinson and M. Cox, "Discrete wavelet analysis of power system transients," IEEE Transactions on Power Systems, vol. 11, no. 4, pp. 2038-2044, Nov 1996.

[23] F. B. Costa, B. A. Souza, and N. S. D. Brito, "Real-time detection of fault-induced transients in transmission lines," Electronics Letters, vol. 46, no. 11, pp. 753-755, May 2010.

[24] F. B. Costa, B. A. Souza, and N. S. D. Brito, "Effects of the fault inception angle in fault-induced transients," IET Generation, Transmission \& Distribution, vol. 6, no. 5, pp. 463-471, May 2012.

[25] F. V. Lopes, D. Fernandes, and W. L. A. Neves, "A traveling-wave detection method based on park's transformation for fault locators," IEEE Transactions on Power Delivery, vol. 28, no. 3, pp. 1626-1634, July 2013.

[26] R. P. Medeiros, F. B. Costa, and K. M. Silva, "Power transformer differential protection using the boundary discrete wavelet transform," IEEE Transactions on Power Delivery, vol. 31, no. 5, pp. 2083-2095, Oct 2016.

[27] EMTP Reference Models for Transmission Line Relay Testing, IEEE Power System Relaying Committee, 2004.

[28] SEL-T400L Time-Domain Line Protection, Schweitzer Engineering Laboratories, Inc., Dez. 2016.

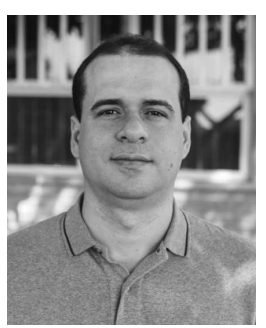

Kleber M. Silva (M'05) received the B.Sc., M.Sc., and $\mathrm{Ph} . \mathrm{D}$. degrees in electrical engineering from University of Campina Grande (UFCG) in 2004, 2005 , and 2009, respectively. Since 2009, he is an Assistant Professor at the University of Braslia (UnB) and the head of the Power System Protection Group. His research interests focus on power system protection, fault location and electromagnetic transients.

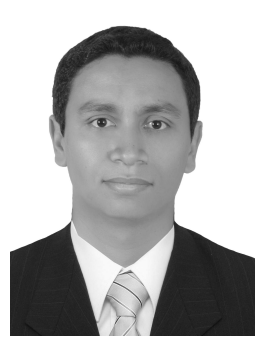

Alfredo Miguel P. Escudero received his B.Sc. degree in Electrical Engineering from National University of Colombia (UNAL), Medellín, Colombia, in 2009. In 2016, he received the master degree in electrical engineering from the University of Brasília (UnB), Brazil. His research interests are mainly focused in power system protection, power quality, generation and electromagnetic transients.

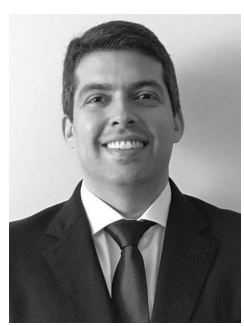

Felipe V. Lopes (M'10) was born in Brazil, 1985. He received his B.Sc., M.Sc. and Ph.D. degrees in Electrical Engineering from Federal University of Campina Grande (UFCG), Brazil, in 2009, 2011 and 2014, respectively. He is currently an Adjunct Professor at the Department of Electrical Engineering at the University of Brasília (UnB), Brazil. His research interests are electromagnetic transients, fault location, power system protection and real-time simulations.

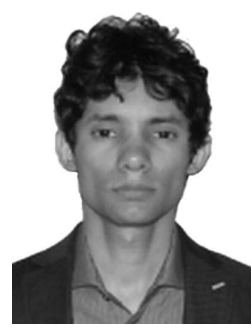

Flavio B. Costa (S'05-M'10) was born in Brazil, 1978. He received the B.Sc., M.Sc., and Ph.D. degrees in Electrical Engineering from UFCG, Brazil, in 2005, 2006, and 2010, respectively. Currently, he is a Professor at Federal University of Rio Grande do Norte (UFRN), Brazil. His research interests include power system protection, electric power quality, renewable energy systems, as well as smart-grid solutions. 